\title{
Helping Families Cope with the Severe Stress of Dravet Syndrome
}

\author{
Peter Camfield, Carol Camfield, Kathleen Nolan
}

\begin{abstract}
A child with Dravet syndrome shakes family life to the core. Dravet syndrome usually has three phases: (1) up to 1-11/2 years: with episodes of febrile status epilepticus but normal development; (2) age 11/2 to 6-10 years: with frequent seizures of varying types, developmental stagnation, behavioural and sleep problems; (3) after 10 years: improvement in seizures, deteriorating gait, intellectual disability but some developmental gains. Complete seizure control is rare-simply prescribing medication is inadequate to help families. Based on structured interviews with 24 families and confirmed by more informal discussions with other families, we suggest strategies for coping with this catastrophe. A child with Dravet syndrome usually means that one parent cannot work-financial pressures should be anticipated. In Stage 1, the approach to status should include a written protocol. An indwelling catheter for rapid venous access may be helpful. In Stage 2, assistance finding qualified babysitters is required, and the extended family needs encouragement to help. Appropriate equipment, rescue medication and protocols should travel with the child. Siblings may benefit from a system of one parent "on call." An internet support group provides an invaluable lifeline. In Stage 3, family isolation may be extreme-respite care and personal time for parents are important. Death from status, accidents and SUDEP (sudden unexplained death in epilepsy) occurs in 15\%. Fear of SUDEP needs to be addressed. Moving from paediatric to adult care is frightening; an epilepsy transition clinic is useful. Attention to these realities may improve the quality of life for both child and family.
\end{abstract}

Keywords: Dravet syndrome, family, help, management, support

doi:10.1017/cjn.2016.248

Can J Neurol Sci. 2016; 43: S9-S12

\section{INTRODUCTION}

From the first episode of status epilepticus, families with a child with Dravet syndrome face enormous challenges that will persist throughout the life of that child (Table 1). Most will face not only their child's persistent seizures but also intellectual disability (often severe), hyperactivity and other neuropsychiatric disorders, disordered sleep and dependency for most activities of daily living. Fear of SUDEP (sudden unexplained death in epilepsy), social isolation, loss of employment, issues of childcare and many additional stressors are common but not easily avoided. While seizure control is an important but elusive goal, physicians and other healthcare professionals can and should assist families with the many other stresses to improve quality of life for the child and the family. ${ }^{1-4}$

This paper outlines areas of family stress and possible interventions through the three stages of Dravet syndrome. The first stage begins in the first year of life, usually before six months, with episodes of febrile status epilepticus, but the child's development remains normal. The second stage starts between one and two years of age, with the onset of multiple seizure types, including myoclonus, shorter afebrile generalized tonic-clonic seizures, atypical absence and dyscognitive (complex partial) seizures. Episodes of convulsive status epilepticus continue along with episodes of non-convulsive status. Developmental progress slows and stagnates. Most, but not all, patients enter a third stage between 5 and 10 years of age that continues throughout the child's life, with fewer seizures, fewer episodes of status and even some developmental gains; for the majority, though, cognitive abilities remain in the range of mild-to-severe intellectual disability.

\section{METHODS}

We have had the privilege of interviewing 24 families with children with Dravet syndrome using a semi-structured interview for a formal study of the impact of Dravet syndrome. ${ }^{3}$ The methodology of our study is outlined in detail elsewhere. ${ }^{3}$ The study was based on a convenience sample from our clinical practice $(n=12)$. In addition, we included some families who attended an Epilepsy Foundation Conference in $2005 \quad(n=8)$ and several from an email Dravet support group $(n=4)$. All of these families were interviewed by one of the authors (KN) using a semi-structured interview, where responses were compared to a well-standardized questionnaire (the Impact of Childhood Neurologic Disability Scale [ICND]) that measures the burden of epilepsy on the patient and family in four domains: epilepsy, cognition, behaviour and physical/neurological function. ${ }^{5-}$ Participants described their child's illness, personal experiences, perceptions of their child's healthcare, and coping mechanisms or support, both effective and lacking. For each stage, parents were asked to report spontaneously events or experiences that were particularly positive or negative in caring for their child and positive and negative experiences with the healthcare system. ${ }^{3}$

From the Department of Paediatrics, Dalhousie University, Halifax, Nova Scotia, Canada (PC, CC); IWK Health Centre, Halifax, Nova Scotia, Canada (PC, CC); Department of Paediatrics, McMaster University, Hamilton, Ontario, Canada (KN).

Received February 23, 2016. Final Revisions Submitted May 3, 2016. Correspondence to: Peter Camfield, IWK Health Centre, 5850 University Avenue, Halifax, Nova Scotia B3K 6R8, Canada. Email: Camfield@dal.ca. 


\section{Table 1: Summary of Special Issues for Children with Dravet Syndrome and Their Families}

\begin{tabular}{l}
\hline All three stages: Finances (only one parent can work) \\
\hline Respite care (everyone too anxious) \\
\hline Fever control (fever strongly provokes seizures) \\
\hline Home care for status epilepticus (rescue benzodiazepines) \\
\hline Stage 1: Diagnosis (clinical evolution and SCN1A mutations) \\
\hline Care for siblings during status epilepticus (keeping them out of the \\
emergency department) \\
\hline Venous access \\
\hline Control of fever \\
\hline Stage 2 Quality of life for siblings ("parent on call") \\
\hline Social isolation, especially from family and grandparents \\
\hline Protocol for management of status epilepticus (for all settings, signed) \\
\hline Control of environmental temperature (air conditioning) \\
\hline Emergency care package (status cannot be predicted, emergency visits are long) \\
\hline SUDEP and sleep monitors \\
\hline School (personal, trained assistant) \\
\hline Stage 3 Gait problems (walking may be lost, levodopa helps some) \\
\hline Care for large, cognitively impaired child with decreased mobility \\
\hline Increased social isolation \\
\hline Transition to adult care \\
\hline Legal guardianship \\
\hline "Well known by no one" (involvement of younger family and friends) \\
\hline tong
\end{tabular}

Subsequent to this study, we have had the opportunity to discuss these issues with other families with a child with Dravet syndrome who we encountered in clinical practice and at two annual meetings of the Dravet Society. It is our opinion that these less formal encounters echoed the results from the more formal study. Many of the interviews were then summarized in a second manuscript. ${ }^{4}$ In the present paper, we have attempted to summarize the stresses, problems and solutions that these families have identified. We are thankful for the families' willingness to share their experiences.

\section{RESUlTS}

\section{Stage 1}

Episodes of status epilepticus are extremely frightening and disruptive to family life. Because these episodes start in the febrile seizure age bracket, they may not be initially appreciated as anything other than prolonged febrile seizures. Most prolonged febrile seizures are relatively innocent over the long run and are characterized by low rates of eventual epilepsy and normal cognition. But as these episodes of status repeat, the family's alarm increases. Fever control becomes an overwhelming preoccupation. It becomes nearly impossible to find childcare, a problem that will likely persist throughout the course of the child's life. Families report great anguish when there is no diagnosis. It is unclear if an early diagnosis changes the medical outcome; however, an early diagnosis can help families to organize their lives around the knowledge that their child will have a persistent problem. Prior to identification of SCN1A mutations as the cause of Dravet syndrome, it was very difficult to make a definite diagnosis before the second stage. We suggest that SCN1A mutation analysis be carried out when there have been two episodes of febrile status during the first year of life. While other epilepsy syndromes are associated with SCN1A mutations, the combination of this mutation with several episodes of febrile status in the first year of life make a compelling case for the diagnosis of Dravet syndrome. ${ }^{6}$ As soon as the diagnosis is made, it is most appropriate to refer the family to one of several online Dravet support groups. These groups provide up-to-date information for parents and assist families in coming to grips with a difficult reality (visit Dravetfoundation.org for a comprehensive listing). In retrospect, parents found the first stage to be the most difficult of the three. ${ }^{3}$

An important aspect of care during Stage 1 and throughout Stages 2 and 3 is temperature control. Fever is a major precipitant of seizures in Dravet syndrome. Even a minor increase in body temperature may precipitate seizures. ${ }^{7}$ Families need to know how to use a thermometer and the optimal dose of antipyretics even though there is no systematic evidence that antipyretic treatment is effective. These children should receive all routine immunizations on schedule to decrease serious contagious infections and further febrile episodes.

A more complex consideration is the role of ambient temperature. In our experience, families are convinced that hot weather, a hot bath or heavy activity with attendant increases in production of body heat may precipitate seizures. Many families report that home air conditioning is a necessity. While home air conditioning has not been subject to a randomised trial, the apparent consensus of its utility makes it a reasonable intervention to be supported by the physician.

In addition, a percutaneous venous access device may be justified to help deliver prompt medication during status epilepticus, particularly in small, pudgy children. ${ }^{8}$

\section{Stage 2}

As other seizure types emerge, seizure control remains a big concern for families, but other issues become very important. Childcare now becomes a crisis, and it is very unusual for both parents to be able to continue to be employed. Assistance with financial issues is important and may require the intervention of a social worker and a financial planner. Sadly, grandparents and other family members often shy away at this stage and will rarely, if ever, agree to care for the child by themselves, especially overnight. Direct explanations to the grandparents by physicians may be helpful. Friends become less engaged, and serious social isolation becomes a reality for the parents. With the many trips to the emergency department, it gradually becomes clear that medical professionals are family friends. Sometimes an emergency room or intensive care nurse will agree to provide some respite care for the family_as they are competent and not intimidated by the child's problem. Physicians should encourage these relationships.

Avoiding trips to an emergency room is an important goal of treatment. All families need to learn how to deliver home "rescue" benzodiazepines. It would appear that midazolam (especially buccal midazolam) is more effective than rectal diazepam. ${ }^{9-11}$ For each child, it soon becomes apparent which treatments are most 
effective to stop status. A challenge for parents is to make sure that physicians follow this information. An "official" protocol detailing the most effective status treatment should be written down and signed by the attending neurologist. Copies should be forwarded to the closest emergency departments and ambulance services. Parents need their own copy in case they are located in another geographic area when status begins. Obviously, this protocol needs to be updated on a regular basis, and an arrogant physician who does not follow the protocol should be approached directly by the treating neurologist.

Siblings of a child with Dravet syndrome face many issues. They are unlikely to understand what is happening and may have significant jealousy issues over the attention attracted by their sibling. Episodes of status interrupt time with their parents. One family arranged an ingenious solution: each day, one parent was "on call" for the child with Dravet syndrome and would go to the emergency room if needed or stay at home to tend to multiple seizures while the other parent would continue activities with the unaffected sibling(s).

In addition, when an ambulance is called, the unaffected sibling may do well to have a specific task identified, such as bringing the "care package" to the front door (see below). Emergency childcare arrangements are also needed for the sibling(s) - a willing neighbour may be of great help. It is highly inappropriate for siblings to accompany the family to an emergency room. These visits are typically very long, with the attendant sleep deprivation, and there is the opportunity in the waiting room to acquire infections that may be later transmitted to the child with Dravet syndrome. On the positive side, parents often noted that the sibling grew up to be especially caring with an awareness of people's feelings and an interest in helping others. ${ }^{3}$

Families need help to develop an emergency "care package" for any ventures outside the home because there is no way to reliably predict the onset of status. This kit includes rescue medication, the status protocol, a cellphone, some money and snacks, plus reading material for the parent in anticipation of a long stay in the emergency department. If the outing involves a stroller for the child, then a locking system is a good idea if an ambulance is needed and the stroller must be left behind. Some families have also included oxygen and a suction machine in their emergency kits.

Looming over Stages 2 and 3 is the issue of SUDEP. About $15 \%$ of patients with Dravet syndrome will die by the time of young adulthood from status epilepticus, accidents or SUDEP. ${ }^{12,13}$ The exact incidence of SUDEP in Dravet syndrome is unclear but is elevated well above that in most other epilepsy syndromes. The incidence may be as high as $10 \%$, with age of death varying from a few months to early adulthood. The mechanism of SUDEP is generally unknown, but in Dravet syndrome there is special concern about cardiac arrhythmia resulting from $S C N 1 A$ mutations in the heart. ${ }^{14}$ SUDEP typically occurs in sleep, and, with this knowledge, families are quick to raise the question of nighttime monitoring. While there is no evidence that monitoring prevents SUDEP, the request is not to be taken lightly. We are not enthusiastic supporters of sleep monitors for children with Dravet syndrome, especially those that only monitor movement; however, a "baby monitor" for the sounds of a seizure at night may provide parents with some comfort. An oxygen saturation monitor may also be considered. One medical professional parent explained to us that she was terrified to go into her child's bedroom every morning for fear of finding her dead, and so a monitor was critical for the sake of her own sleep.

School begins during Stage 2. Children with Dravet syndrome have a right to attend school, but they must have a one-on-one teacher assistant who has been specially trained about seizures. Physicians should play a role in designing the "seizure" curriculum for the teacher assistant with attention to obvious seizures and convulsive status but also to non-convulsive status. A detailed school protocol for dealing with status is needed. Because emergency visits are common, a parent must continue to be available during school hours.

\section{Stage 3}

Not all children with Dravet syndrome enter the third, calmer stage beginning at about age 5-10 years. Seizures become less frequent, although generalized tonic-clonic seizures during sleep frequently persist and very few patients become completely seizure-free. Episodes of status epilepticus are much less common, giving some stability to family life that permits easier access to babysitter or respite care. Some improvement in cognitive function is possible; however, difficulties in walking with a characteristic crouching gait often become more obvious and significant. Some children have improvements in their gait with levodopa; however, others become completely unable to walk, which further increases their dependency. ${ }^{15} \mathrm{~A}$ new challenge for the family is caring for a larger, immobile and cognitively impaired child with limited mobility. A wheelchair, bathroom and other house renovations, lifting devices and wheelchair transportation may be needed. Guidance is needed from occupational therapy, physiotherapy and social work, the latter because the equipment is extremely expensive. Parents report that they are less stressed at this point but feel increasingly socially isolated. They likely benefit from encouragement to pursue their own interests that have been on hold for many years. In particular, rekindling previous friendships seems important.

The next predictable crisis is the move to adult medical care. The relationship between parents and paediatric caretakers is intense, with parents developing a great deal of trust and confidence in their paediatric care team. ${ }^{16,17}$ The change to an adult medical system is very frightening, particularly if epilepsy remains very active and episodes of status are still common. The paediatric team may fear that adult care will not be complete. Multidisciplinary clinics and ancillary services are often less available in adult medicine, so that care is likely to be fragmented. A transition clinic can be very helpful, where the patient and family are seen for several visits jointly by the new adult neurologist together with the paediatric neurologist. ${ }^{18}$ Alternately, transition to an adult center that specializes in rare epilepsies may also be very successful. ${ }^{19}$ Drugs such as stiripentol may be unfamiliar to adult neurologists, and the paediatric approach to status for some Dravet patients may be quite unconventional. Adult emergency room physicians need to become involved in the transition process, and other ancillary services should be engaged. The family physician may not have played a significant role during the childhood years but now, with some encouragement, can provide a "medical home." 20

Transition also needs to address guardianship (a formal legal process) to establish who makes decisions for the now young adult with Dravet syndrome. While life expectancy in Dravet 
syndrome has not been clearly defined, many patients live well into adulthood, when their parents may have died and siblings moved away. It is likely that they will live in some form of institutional setting with limited medical records and frequent staff turnovers, which leads to the Dravet patient becoming "well known by no one.",1 Encouragement for younger family friends and family members to stay involved would seem an additional goal for transition.

\section{Conclusions}

Our summary of the issues in caring for a child with Dravet syndrome is not "objective," although the families' concerns did parallel their responses to a standardized measure of the burden of epilepsy. ${ }^{3,5}$ It appears that every aspect of family life is altered by a child with Dravet syndrome. Physicians can assist families to cope by offering strategic suggestions at each stage of the disorder. While the condition is very stressful, there may be many positives. Children with Dravet syndrome may be very loving and lovable and play an integral role in the lives of their families.

\section{Disclosures}

Peter Camfield has the following disclosure: Biocodex, speaker, honorarium. Carol Camfield and Kathleen Nolan have nothing to disclose.

\section{REFERENCES}

1. Brunklaus A, Dorris L, Zuberi SM. Comorbidities and predictors of health-related quality of life in Dravet syndrome. Epilepsia. 2011;52:1476-82.

2. Strzelczyk A, Schubert-Bast S, Reese JP, Rosenow F, Stephani U, Boor R. Evaluation of health-care utilization in patients with Dravet syndrome and on adjunctive treatment with stiripentol and clobazam. Epilepsy Behav. 2014;34:86-91.

3. Nolan K, Camfield CS, Camfield PR. Coping with Dravet syndrome: parental experiences with catastrophic epilepsy. Dev Med Child Neurol. 2006;48:761-5.

4. Nolan K, Camfield PR, Camfield CS. Coping with a child with Dravet syndrome: insights from families. J Child Neurol. 2008;23:690-4.

5. Camfield C, Breau L, Camfield P. Assessing the impact of pediatric epilepsy and concomitant behavioral, cognitive, and physical/ neurologic disability: impact of the Childhood Neurologic Disability Scale. Dev Med Child Neurol. 2003;45:152-9.

6. Scheffer IE, Zhang YH, Jansen FE, Dibbens L. Dravet syndrome or genetic (generalized) epilepsy with febrile seizures plus? Brain Dev. 2009;31:394-400.
7. Verbeek NE, Wassenaar M, van Campen JS, Sonsma A, Gunning B, Knoers N, et al. Seizure precipitants in Dravet syndrome: what events and activities are specifically provocative compared with other epilepsies? Epilepsy Behav. 2015;47:39-44.

8. Bothwell JE, Dooley JM, Gordon KE, Wood EP. Port-A-Cath use in refractory seizure disorders. Arch Dis Child. 2001;85:510.

9. Camfield CS, Camfield PR, Smith E, Dooley JM. Home use of rectal diazepam to prevent status epilepticus in children with convulsive disorders. Arch Dis Child. 1989;4:125-6.

10. McIntyre J, Robertson S, Norris E, Appleton R, Whitehouse WP, Phillips B, et al. Safety and efficacy of buccal midazolam versus rectal diazepam for emergency treatment of seizures in children: a randomised controlled trial. Lancet. 2005; 366(9481):205-10.

11. Brigo F, Nardone R, Tezzon F, Trinka E. Nonintravenous midazolam versus intravenous or rectal diazepam for the treatment of early status epilepticus: a systematic review with meta-analysis. Epilepsy Behav. 2015;49:325-36.

12. Sakauchi M, Oguni H, Kato I, Osawa M, Hirose S, Kaneko S, et al. Retrospective multi-institutional study of the prevalence of early death in Dravet syndrome. Epilepsia. 2011;52:1144-9.

13. Skluzacek JV, Watts KP, Parsy O, Wical B, Camfield P. Dravet syndrome and parent associations: the IDEA League experience with comorbid conditions, mortality, management, adaptation, and grief. Epilepsia. 2011;52(Suppl 2):95-101.

14. Auerbach DS, Jones J, Clawson BC, Offord J, Lenk GM, Ogiwara I, et al. Altered cardiac electrophysiology and SUDEP in a model of Dravet syndrome. PLoS One. 2013;8: e77843.

15. Fasano A, Borlot F, Lang AE, Andrade DM. Antecollis and levodopa-responsive parkinsonism are late features of Dravet syndrome. Neurology. 2014;82:2250-1.

16. Camfield P, Camfield C. Transition to adult care for children with chronic neurological disorders. Ann Neurol. 2011;69: 437-44.

17. Scheffer IE, Dravet C. Transition to adult life in the monogenic epilepsies. Epilepsia. 2014; 55(Suppl 3):12-5.

18. Appleton RE, Chadwick D, Sweeney A. Managing the teenager with epilepsy: paediatric to adult care. Seizure. 1997;6:27-30.

19. Kuchenbuch M, Chemaly N, Chiron C, Dulac O, Nabbout R. Transition and transfer from pediatric to adult health care in epilepsy: a family survey on Dravet syndrome. Epilepsy Behav. 2013;29:161-5.

20. American Academy of Pediatrics, American Academy of Family Physicians, American College of Physicians, Transitions Clinical Report Authoring Group, Cooley WC, Sagerman PJ. Supporting the health care transition from adolescence to adulthood in the medical home. Pediatrics. 2011;128:182-200.

21. Bigby C. Known well by no-one: trends in the informal social networks of middle-aged and older people with intellectual disability five years after moving to the community. J Intellect Dev Disabil. 2008;33:148-57. 